\title{
Habilidades de lectura de jóvenes al término de la secundaria: ¿qué se predice para su lectura en contextos universitarios?'
}

\author{
Reading Skills of Youth at the End of Secondary Education: \\ What is Predicted for their Reading in University Contexts?
}

\author{
MARCELA CABRERA POMMIEZ \\ Universidad de Las Américas \\ mcabrerap@udla.cl \\ SERGIO CARUMAN JORQUERA \\ Universidad de Chile \\ Chile \\ scaruman@uchile.cl
}

(Recibido: 2O/II/2OI8; aceptado: 27/04/20I9)

Resumen. Se presentan los resultados y conclusiones derivados de un estudio realizado sobre un corpus de datos provenientes de la prueba estandarizada usada en Chile para seleccionar a los estudiantes que quieren acceder al nivel universitario, denominada Prueba de Selección Universitaria-Lenguaje. Los datos corresponden a ocho aplicaciones, periodo 2005 a 2012. El corpus está compuesto por tres elementos: un grupo de textos estímulo (expositivos y argumentativos, cuyas características discursivas permiten asimilarlos a textos académicos), los ítems asociados a cada texto, más los estadígrafos (dificultad y omisión) que dan cuenta de cuántos estudiantes respondieron la clave y cuántos omitieron. El análisis de esta información permitió constatar que los egresados de educación media presentan un bajo nivel de comprensión lectora, especialmente en el procesamiento de la superficie textual para obtener información implícita, tal como: interpretación de la perspectiva discursiva que adopta el emisor en el desarrollo del tema; reconocimiento de la organización interna en un texto expositivo o identificación de la tesis que se plantea en un texto argumentativo. Estos resultados son coincidentes con otros estudios que evidencian dificultades en lectura comprensiva de jóvenes que han terminado su educación secundaria.

Palabras clave: comprensión lectora; prueba de lectura; lectura académica.
Abstract. This paper presents results and conclusions derived from a study conducted on a corpus of data from the standardized test used in Chile to select students who want to access college. This test is called the University Selection Test-Language. The data correspond to eight applications, period 2005 to 2012 . The corpus is composed of three elements: a group of stimulus texts (expository and argumentative, whose discursive characteristics allow them to be assimilated to academic texts), the items associated with each text, plus the statisticians (difficulty and omission) that account for how many students answered the key and how many omitted. The analysis of this information allows for the verification that the graduates of secondary education present a low level of reading comprehension, especially in the processing of the textual surface to obtain implicit information, such as: interpretation of the discursive perspective adopted by the issuer in the development of the topic; recognition of the internal organization in an expository text or identification of the thesis that arises in an argumentative text. These results are consistent with other research that shows difficulties in reading comprehensively for young people who have finished their secondary education.

Keywords: reading comprehension; reading test; academic reading.

\footnotetext{
${ }^{\text {I }}$ Para citar este artículo: Cabrera Pommiez, Marcela y Caruman Jorquera, Sergio (2O2O). Habilidades de lectura de jóvenes al término de la secundaria: ¿qué se predice para su lectura en contextos universitarios? Alabe 21. [www.revistaalabe.com] DOI: I0.15645/Alabe2020.2I.II
} 


\section{Introducción}

El artículo indaga en las habilidades de lectura que poseen los estudiantes chilenos al término de su enseñanza media, a partir de la información que puede extraerse de ocho aplicaciones de la Prueba de Selección Universitaria-Lenguaje y Comunicación (en adelante, PSU-L), elaborada por el Departamento de Medición, Evaluación y Registro Educacional (DEMRE), de la Universidad de Chile, y que cuenta con un universo amplio de estudiantes (ahora cercano a 280 mil) que, año a año, la rinden para obtener un puntaje que les permita continuar estudios universitarios. Esto implica que los egresados de enseñanza media busquen un buen resultado, por lo cual el análisis de esta información es representativo para determinar cómo los jóvenes están leyendo los textos que, se supone, deberían ser capaces de leer comprensivamente después de haber cursado la etapa escolar, dada la inclusión de dichos contenidos y competencias en el currículo de enseñanza básica y media, a lo largo de los doce años prescritos por este.

Algunas investigaciones (Neira et al., 20I5; Roldán y Zabaleta, 2OI6) dan cuenta de carencias en este sentido, detectadas tanto en estudiantes de enseñanza secundaria como terciaria. En esta última etapa, las deficiencias en lectura comprensiva de textos académicos cobra especial relevancia, dado que estos son una importante vía de acceso al conocimiento, por lo cual situaciones como reprobación de asignaturas, deserción o escaso avance en la malla curricular se relacionan, en alguna medida, con carencias en habilidades comunicativas vinculadas al discurso académico.

En el artículo se desarrollan algunos conceptos relacionados con el discurso académico; se presenta el modelo de evaluación que organiza el instrumento PSU-L; se analizan algunos ítems que muestran estrategias de lectura y habilidades cognitivas que deberían activarse en los estudiantes para resolverlos adecuadamente; $y$, finalmente, se presenta, como conclusión, una apreciación crítica fundada, en torno a las habilidades de lectura de la población objeto.

\section{Antecedentes}

\section{I. Características del discurso académico}

Hace aproximadamente veinte años, comenzó a hablarse, en el ámbito de la lingüística, la comunicación y la educación (Narvaja de Arnoux et al., I996; Bono y De la Barrera, I998; Escofet, Rubio y Tolchinsky, I999) sobre la situación de formación inicial de los estudiantes que ingresaban a las aulas universitarias: con frecuencia, estos no disponían de las habilidades comunicativas necesarias para leer comprensivamente los textos listados en las bibliografías de las asignaturas, ni de la capacidad para escribir textos satisfactorios en el ámbito académico, los que, además, serían objeto de evaluación por parte de los docentes. Al decir "satisfactorios", debe entenderse que el término aludía -y aún alude- no solo al manejo de contenidos, sino también a la adecuación comunicativa 
de los mismos. A partir de esta situación, paulatinamente fue tomando forma el concepto de discurso académico, definido como el "[...] conjunto de prácticas discursivas que se genera en el ámbito o esfera de la academia, ya que es en este ámbito -la universidad, los institutos superiores y los centros de investigación, entre otros- en donde se ejecutan sus principales funciones" (Núñez y Espejo, 2005: I36). Este tipo particular de discurso se caracteriza por rasgos que han sido bien descritos por la bibliografía y que suponen un conocimiento superior de estrategias comunicativas, a saber:

a) alto nivel de corrección lingüística y adecuación al registro estándar, pues se trata de un tipo de comunicación vinculada al saber, distinta del registro coloquial o periodístico, por ejemplo;

b) uso de léxico preciso y especializado;

c) alusiones a procesos y situaciones abstractos más que a acciones concretas (Marín, 2006);

d) períodos sintácticos extensos y oraciones complejas;

e) presencia de un emisor que es experto en la materia y que se comunica para difundir, refutar, ampliar o complementar el conocimiento existente en torno a un tema específico, inserto en una disciplina;

f) un grado elevado de objetividad o neutralidad, aunque no total, pues necesariamente el emisor adopta un posicionamiento con respecto al contenido de su discurso (Bolívar, Beke y Shiro, 2OIO), el que se conjuga con un propósito argumentativo de fondo, en el sentido de que el emisor busca convencer acerca de la utilidad y/o veracidad de lo que escribe o habla;

g) manejo de ciertos géneros orales y escritos (artículo de investigación, póster, ponencia, ensayo, reseña, entre otros), que deben ser aprendidos por los estudiantes nóveles, dentro de sus disciplinas, en lo que Carlino (2013) denomina proceso de enculturación, que consiste en incorporarse comunicativamente a sus comunidades discursivas, las que poseen prácticas discursivas situadas;

h) la lectura y escritura de los géneros académicos arriba mencionados requiere el dominio de las características y estructura de dos secuencias textuales en particular: la exposición y la argumentación. Ello implica la utilización, a lo largo del texto, de estrategias y recursos discursivos como: definición, explicación, descripción, enumeración, clasificación, para el texto expositivo -o explicativo, como también se ha llamado (Álvarez, I996)-; y planteamiento de tesis, exposición de argumentos y contraargumentos, presentación de respaldos, ejemplificación y polifonía (citación), entre otros, para el texto argumentativo (Bassols y Torrent, 2OI2; Calsamiglia y Tusón, 2OI2).

Todas estas características provocan que el discurso académico sea un tipo de comunicación difícil no solo por la necesaria vinculación con el saber disciplinario, sino porque requiere el desarrollo de prácticas de lectura y escritura intencionadas para que pueda ser adquirido. La situación de la mayoría de los estudiantes, frente a este tipo de 
requerimiento discursivo superior, es de analfabetismo académico, descrito por Hall y López (2OII, p.I68) en los siguientes términos:

En las últimas décadas, se ha acuñado el término analfabetismo académico para referirse a las dificultades que presentan los estudiantes que cursan estudios superiores cuando interpretan y producen textos académicos. Publicaciones recientes coinciden en que la lectura y la escritura requieren el desarrollo de competencias complejas que no han sido adquiridas aún por los estudiantes cuando ingresan a sus estudios universitarios.

La respuesta de las instituciones de educación superior ante el analfabetismo académico de sus estudiantes es la alfabetización académica -traducción del inglés literacy (Braslavsky, 2003) y hoy denominada también literacidad (Escarpeta, 20i9) o letrismo académico (Sánchez, 2OI6), concepto que: "Señala el conjunto de nociones y estrategias necesarias para participar en la cultura discursiva de las disciplinas así como en las actividades de producción y análisis de textos requeridas para aprender en la universidad" (Carlino, 2005: I3). Actualmente, existe consenso en torno a la idea de que este proceso no debe realizarse mediante intervenciones aisladas, usualmente concentradas en primer año, sino que a lo largo del currículo y en estrecha vinculación con este (Carlino, 2OI3; Moyano y Giudice, 20I6).

Entonces, existe un desfase entre las habilidades comunicativas, el manejo discursivo y el conocimiento lingüístico de los jóvenes que egresan de la educación secundaria y se enfrentan a la lectura y la escritura en el nivel terciario; situación que genera tareas tanto para el sistema escolar (mejorar este desfase comunicativo y preparar adecuadamente a los estudiantes para su participación en la sociedad) como para el superior (promover la alfabetización académica, facilitando así la principal vía de acceso y producción del conocimiento).

\subsection{La comprensión lectora en pruebas de admisión al sistema universitario}

La PSU es una batería de pruebas vigente en el sistema educacional chileno desde al año 20O3, que reemplazó a la Prueba de Aptitud Académica (1967-2002), pero que mantuvo su función principal: seleccionar, entre los egresados de educación media, a aquellos que pueden acceder a una carrera en una institución de educación superior, en virtud de los resultados obtenidos (traducidos a puntaje), los cuales se ponderan con las calificaciones de los cuatro años de educación media más el puntaje ranking de cada estudiante $^{2}$. Además de su rol en la selección, este instrumento posee referencia curricular, es decir, evalúa los contenidos mínimos obligatorios (CMO) de algunas asignaturas del Plan de Estudios, declarados en el Marco Curricular (MINEDUC, 1998 y 2009), prescritos por ley en la educación chilena.

\footnotetext{
${ }^{2}$ Para más información acerca del proceso de admisión a las universidades chilenas, véase http: //psu.demre.cl/ proceso-admision/ [Consulta: I2-I-2OI9].
} 
La PSU está constituida por dos pruebas obligatorias (Matemática y Lenguaje y Comunicación) y dos electivas (Ciencias y Ciencias Sociales). Para el desarrollo de esta investigación, solo se consideró la prueba de Lenguaje (PSU-L), que, en el periodo 2005 a 2OI2, poseía las siguientes características:

a) Constaba de 8o ítems de selección múltiple con respuesta única, elaborados con cinco opciones.

b) Se articulaba en tres secciones: la sección I evaluaba conocimientos generales de la asignatura Lenguaje y Comunicación, como tipos de mundo narrado o mecanismos de correferencia; la sección II era una evaluación indirecta de la producción escrita, pues presentaba ítems de manejo de conectores y marcadores textuales, más ítems de ordenación de enunciados; la sección III estaba constituida por 50 ítems de comprensión de lectura, I5 de vocabulario contextual y 35 de comprensión, referidas a diversos textos estímulo, tanto literarios como no literarios.

c) La sección III evaluaba habilidades de comprensión lectora de los estudiantes vinculándolas con los contenidos de la asignatura, es decir, junto a la comprensión como proceso cognitivo que otorga un sentido a la información textual, se consideraban contenidos curriculares en la elaboración de ítems. Por ejemplo, tareas de lectura referidas al discurso expositivo se orientaban en virtud de los CMO de II medio, así como ítems basados en narrativa contemporánea tomaban elementos de análisis definidos en el Marco Curricular para IV medio.

d) El modelo de evaluación de la comprensión lectora definía tres grandes competencias de lectura y, dentro de cada competencia, habilidades cognitivas, las que se ordenaban desde la recuperación de información explícita hasta la evaluación de aspectos de fondo y forma del texto y consideraban tareas de lectura como identificación, síntesis, inferencia, interpretación y evaluación de la información textual.

En Cabrera y Caruman (20I8: IO9-II5) se puede ver una presentación exhaustiva acerca de temas como: clasificación textual, concepto y competencias de lectura, modelo de evaluación de la comprensión lectora en pruebas estandarizadas y análisis de ítems. 


\section{Análisis de textos y preguntas PSU-Lenguaje}

La investigación realizada corresponde a un diseño no experimental, ex post facto y descriptivo (Bisquerra, 20I4), pues consiste en el análisis de datos provenientes de ocho aplicaciones (2OO5-2OI2) de un instrumento estandarizado (PSU-L), que permite describir las habilidades de lectura de una población que ha terminado la enseñanza secundaria y aspira a acceder a la educación superior. El análisis es de tipo mixto, pues considera datos cuantitativos (porcentaje de dificultad y de omisión de cada ítem) y cualitativos (comentario de textos y preguntas). Si bien las versiones de pruebas del periodo consideraban un total de ochenta preguntas, en esta investigación se tomaron solo las cincuenta últimas (referidas a comprensión lectora, sección III de la prueba). Dada la importancia de la PSU para el acceso al nivel universitario, cada aplicación reunía a una población superior a los 200.000 mil estudiantes, por lo cual los datos de esta investigación recogen las tendencias de lectura de un $n$ total de r.825·597 jóvenes chilenos.

La metodología consideró dos etapas. La primera estuvo focalizada en los textos usados como estímulo de la comprensión: se revisó la clasificación de cada uno, basada principalmente en tipos textuales (literarios/no literarios), y que fue realizada de acuerdo al currículum vigente en ese momento (Mineduc, 2005 y 2009). Además, se contabilizó cuántos textos había en cada categoría (corpus total), lo cual permitió determinar qué tipos textuales tenían mayor y menor presencia en las pruebas PSU-L; luego se seleccionó el corpus específico, que estuvo constituido por algunos textos clasificados como no literarios-expositivos y argumentativos más literarios-ensayo, cuyas características los hacían asimilables a textos académicos. En la segunda etapa se revisaron los ítems del corpus, con los resultados de su aplicación, para relacionar su comportamiento estadístico con la tarea de lectura asociada, las características del texto estímulo y la estrategia de lectura académica comprensiva que estaba implicada en su resolución.

\section{I. Resultados del análisis de textos}

En la tabla I se presenta la clasificación de la totalidad de textos estímulo usados en las ocho aplicaciones de la PSU-L, más la cantidad de textos que corresponde a cada categoría. 
Tabla 1. Total de textos usados en la PSU-Lenguaje (periodo 2005-2012)

\begin{tabular}{|c|c|c|c|c|c|}
\hline \multicolumn{2}{|c|}{ Textos literarios } & \multicolumn{2}{c|}{ Textos no literarios } \\
\hline Géneros & $\mathbf{n}$ & Porcentaje & Géneros & $\mathbf{n}$ & Porcentaje \\
\hline Narrativos & 17 & $20,1 \%$ & Expositivos & 34 & $40,5 \%$ \\
\hline Líricos & 9 & $10,7 \%$ & Argumentativos & 14 & $16,7 \%$ \\
\hline Dramáticos & 2 & $2,4 \%$ & Dialógicos & 4 & $4,8 \%$ \\
\hline Ensayísticos & 3 & $3,6 \%$ & Discurso público & 1 & $1,2 \%$ \\
\hline Totales parciales & $\mathbf{3 1}$ & $\mathbf{3 6 , 8 \%}$ & & $\mathbf{5 3}$ & $\mathbf{6 3 , 2 \%}$ \\
\hline Total & & & $\mathbf{8 4}$ & & \\
\hline
\end{tabular}

Fuente. Elaboración propia

Se aprecia que la mayor cantidad de textos $(63,2 \%$ del corpus total) corresponde a no literarios. Los tipos textuales que constituyen las primeras tres mayorías en cuanto a número son: expositivos, narrativos y argumentativos (en ese orden), selección que, en gran medida, se explica porque estos tres tipos textuales exhiben buenos estadígrafos en aplicaciones piloto.

Para constituir el corpus de esta investigación, a partir de la totalidad de textos expositivos y argumentativos, se seleccionaron aquellos textos (y sus ítems) que más se acercaban al discurso académico, esto es, que presentaban las siguientes características: adecuación al registro estándar; tema central referido a un ámbito del saber y no necesariamente familiar para los estudiantes; selección léxica correspondiente al área temática; y, por último, géneros discursivos propios de la academia: artículo de investigación, capítulo de texto especializado (o manual), conferencia. Los textos ensayísticos quedaron incluidos de inmediato. En cuanto a los géneros de la prensa escrita, la noticia no se consideró, debido a su brevedad y temática actual; pero sí algunos reportajes. En comparación con los demás textos utilizados en las pruebas (por ejemplo, poemas, fragmentos de cuentos o de obras dramáticas, noticias, columnas de opinión o editoriales de periódi$\cos )$, los textos del corpus presentan un grado más alto de abstracción, una prosa elaborada y marcas textuales de la subjetividad del emisor en función del tema central; sin que pueda hablarse de textos académicos propiamente tales, pues su selección se restringe necesariamente a las capacidades de lectura de jóvenes que han terminado su educación media. En la tabla 2 se presentan algunos ejemplos. 
Tabla 2. Ejemplos de textos del corpus

\begin{tabular}{|c|c|c|c|c|}
\hline Prueba & $\begin{array}{c}\text { Texto } \\
\text { estímulo }\end{array}$ & $\begin{array}{c}\text { Género } \\
\text { discursivo }\end{array}$ & Tema & Referencia \\
\hline 2007 & $\begin{array}{l}\text { Fragmento } \\
\text { expositivo }\end{array}$ & $\begin{array}{c}\text { Texto } \\
\text { especializado } \\
\text { (lingüística) }\end{array}$ & $\begin{array}{c}\text { El valor social del } \\
\text { discurso. }\end{array}$ & $\begin{array}{l}\text { Van Dijk, Teun. 2005. Es- } \\
\text { tructura y funciones del dis- } \\
\text { curso. } 14^{\mathrm{a}} \text { ed. México D.F.: } \\
\text { Siglo XXI Editores. }\end{array}$ \\
\hline 2010 & $\begin{array}{l}\text { Fragmento } \\
\text { expositivo }\end{array}$ & Ensayo & $\begin{array}{l}\text { Análisis } \\
\text { antropológico del } \\
\text { pueblo mexicano. }\end{array}$ & $\begin{array}{l}\text { Paz, Octavio. 1999. “Todos } \\
\text { Santos, día de muertos”. En: } \\
\text { El laberinto de la soledad. } \\
\text { México D.F.: FCE. }\end{array}$ \\
\hline 2011 & $\begin{array}{c}\text { Fragmento } \\
\text { argumentativo }\end{array}$ & Ensayo & $\begin{array}{l}\text { El poder de la TV } \\
\text { en la educación } \\
\text { infantil. }\end{array}$ & $\begin{array}{l}\text { Savater, Fernando. 1997. El } \\
\text { valor de educar. Barcelona: } \\
\text { Ariel. }\end{array}$ \\
\hline 2012 & $\begin{array}{l}\text { Fragmento } \\
\text { expositivo }\end{array}$ & $\begin{array}{c}\text { Capítulo de } \\
\text { texto } \\
\text { especializado }\end{array}$ & $\begin{array}{l}\text { Similitudes y } \\
\text { diferencias entre la } \\
\text { ciencia y el arte en } \\
\text { cuanto a la } \\
\text { transformación del } \\
\text { conocimiento. }\end{array}$ & $\begin{array}{c}\text { Díaz, José Luis. 2002. "Cien- } \\
\text { cia y arte: la inteligencia de } \\
\text { las musas". En: El ábaco, la } \\
\text { lira y la rosa. Las regiones } \\
\text { del conocimiento. México } \\
\text { D.F.: Fondo de Cultura Eco- } \\
\text { nómica }\end{array}$ \\
\hline
\end{tabular}

Fuente: elaboración propia.

\subsection{Resultados del análisis de ítems}

A continuación, se presenta el análisis de algunos fragmentos de textos estímulo más los ítems que evalúan la comprensión de recursos discursivos propios de textos académicos. Los dos estadígrafos utilizados para este análisis (dificultad y omisión) expresan los valores que adquieren los ítemes en las aplicaciones oficiales de las pruebas; en el caso de la dificultad, en la Teoría Clásica, este valor se representa porcentualmente, en una escala del o (muy difícil) al ıoo\% (muy fácil), dado que el constructo está basado en la cantidad total de estudiantes que rinden la prueba. Si el Ioo\% responde correctamente, la pregunta es demasiado fácil; si el o\% no puede responderla, la pregunta es inabordable. La omisión, en cambio, expresa el porcentaje de estudiantes que prefieren no responder la pregunta, pues el constructo de este período (2006-20I2) todavía descontaba o.25 por respuesta errónea del puntaje bruto obtenido por cada estudiante. En el caso de la prueba de Lenguaje, valores porcentuales superiores al 3o\% determinan que se trata de un ítem con alta omisión. Ambos valores, además, pueden correlacionarse estadísticamente en ciertos casos (Aiken, I99I, Crocker y Algina, I986). 


\section{Ejemplo I: clasificación temática de un texto}

Texto estímulo: fragmento de Apología de Sócrates (Platón), 49I palabras, prueba 2010 , texto $9^{3}$. Sócrates se dirige a sus jueces tratando de levantar una defensa de su persona y desestimando las acusaciones hechas en su contra.

La pregunta 65 corresponde a la habilidad de identificar y solicita al estudiante que, una vez leído el fragmento, lo clasifique de acuerdo a su temática.

65. De acuerdo con la naturaleza de los argumentos utilizados por el emisor, se puede afirmar que el texto es de tipo
A) ético-filosófico
B) jurídico- filosófico.
C) político-social.
D) literario-filosófico.
E) político-moral.

Clave: A

El ítem presenta una dificultad de I $4,9 \%$ y una omisión de $45,3 \%$, ambas bastante altas, lo que demuestra que resultó difícil de abordar, situación que puede atribuirse a una característica morfosintáctica de las opciones: están elaboradas como palabras compuestas en vez de simples, por lo cual el estudiante debe conjugar, en su selección, dos ámbitos temáticos. La opción B resultó un distractor atractivo, elegido por el 20,4\% de la población, lo que se explica porque el discurso de Sócrates se desarrolla en el contexto de un proceso judicial; sin embargo, sus argumentos tienen una naturaleza ética y filosófica, dado que se refiere principalmente a la justicia, a la verdad y su actuación como orador.

\section{Ejemplo 2: identificación de tesis en un texto argumentativo}

Texto estímulo: fragmento de "Razones del corazón. La educación del deseo" (Adela Cortina), $3^{8} 5$ palabras, prueba 2007, texto $4_{4}^{4}$. Es un texto argumentativo donde se desarrolla la idea de que la inteligencia emocional es un factor relevante en el desarrollo personal de cada individuo y en su relación con el mundo y con el resto de las personas.

La pregunta 8o evalúa la capacidad del estudiante para extraer una de las tesis que se presentan en el fragmento leído, para lo cual debe descomponer el texto y asignar funciones a los párrafos, en virtud de la estructura del texto argumentativo (premisatesis-argumentos-conclusión).

\footnotetext{
3 Ver texto completo y preguntas en "Pruebas de Matemática y Lenguaje y Comunicación”, página I3, disponible en: http://www.psu.demre.cl/publicaciones/listado-2OI2 [Consulta 22-OI-2OI9].

4 Ver texto y preguntas en "Facsímil de Lenguaje y Comunicación”, página 43, disponible en: https://psu.demre. $\mathrm{cl} /$ publicaciones/listado-2009 [Consulta 22-OI-2OI9].
} 
80. Una de las tesis que el fragmento anterior presenta es que

A) nuestra conducta moral aparece regulada por nuestros sentimientos y emociones.

B) el "saber hacer" de los humanos es una capacidad sustancialmente afectiva y solo accidentalmente racional.

C) un adecuado control racional de nuestras emociones es necesario para interactuar eficazmente con la realidad.

D) el desinterés de algunas personas por la realidad que los rodea se explica por una sobreabundancia de emociones.

E) nuestra percepción de la realidad está mediatizada y determinada por nuestros sentimientos y emociones.

Clave: E

Este ítem fue resuelto correctamente por el $26,4 \%$ de los estudiantes y omitido por el $36,9 \%$ de estos, es decir, se aprecia que tanto la dificultad como la omisión son altas. Las cifras precedentes muestran que las habilidades de lectura de textos argumentativos no están bien desarrolladas en la población, pues la determinación de la tesis y los argumentos que la sustentan es un procedimiento necesario para su adecuada comprensión, el cual se complica por el hecho de que, a menudo, se trata de información implícita en el texto, que debe extraerse mediante análisis e interpretación de la superficie textual.

\section{Ejemplo 3: determinación de patrón de organización textual en una secuencia ex- positiva}

Texto estímulo: reportaje de diario El Mercurio sobre pinturas rupestres, $249 \mathrm{pa}-$ labras, prueba 2007 , texto ${ }^{2} 2^{5}$. Se trata de un fragmento expositivo donde se presentan tres momentos de descubrimiento del primitivo arte rupestre, momentos que se encuentran explícitamente señalados en la superficie textual a través de los siguientes marcadores discursivos: "En los años 5o" (primer párrafo), "En las décadas siguientes" y "recientemente" (segundo párrafo).

El ítem 68 corresponde a la habilidad de comprender-analizar y evalúa la identificación del patrón textual propio de la exposición de información que se utilizó en el fragmento.

68. El modelo de organización textual que sigue el fragmento es de

A) problema - solución.

B) secuencia temporal.

C) enumeración descriptiva.

D) causa - consecuencia.

E) descripción subjetiva.

Clave: B

5 Texto y preguntas ídem documento anterior, páginas $3^{8}$ y 39. 
La clave es "secuencia temporal", pues el texto entrega información sobre las pinturas rupestres descubiertas en la zona norte de Chile a partir del establecimiento de una cronología en tres etapas, desde lo más antiguo a lo más reciente, cada una de las cuales está asociada a determinados hechos. Este ítem presentó una dificultad media (40,8\%) y una omisión alta $(36,4 \%)$.

\section{Ejemplo 4: reconocimiento de un prejuicio del emisor en la presentación de argu- mentos}

Texto estímulo: fragmento de El valor de educar (Fernando Savater), 5 IO palabras, prueba 20II, texto $6^{6}$. Se trata de un texto argumentativo (ensayo), donde se plantea que la televisión constituye una poderosa influencia para la mente infantil, pues presenta de manera directa y cruda aspectos de la humanidad (como el sexo y la violencia), que los niños antes descubrían paulatinamente. Se postula una visión negativa de la televisión, al mostrarla como herramienta de conocimiento demasiado explícito, que destruye la inocencia infantil.

En el ítem 59 se solicita el reconocimiento de un prejuicio por parte del emisor, el cual aparece en las primeras líneas del fragmento: “La revolución que la televisión causa en la familia, sobre todo por su influencia en los niños, nada tiene que ver con la perversidad bien sabida de sus contenidos sino que proviene de su eficacia como instrumento para comunicar conocimientos"; es decir, se da por hecho que la información entregada por la televisión es perversa y todo el mundo lo sabe. En el ítem se presentan varias citas textuales, para que el estudiante reconozca en cuál se expresa un prejuicio del emisor, es decir, una opinión negativa sin fundamento demostrado.

59. ¿En cuál de las siguientes opciones aparece un prejuicio expuesto en el texto con relación al tema de la televisión?

A) "La revolución que la televisión causa en la familia, sobre todo por su influencia en los niños, nada tiene que ver con la perversidad bien sabida de sus contenidos (...)"

B) "Durante siglos, la infancia se ha mantenido en un limbo aparte del que solo iban saliendo gradualmente los pequeños de acuerdo con la voluntad pedagógica (...)"

C) "Los modelos de conducta y de interpretación del mundo que se ofrecían al niño no podían ser elegidos voluntariamente (...)"

D) "La identidad infantil (...) consistía en ignorar esas cosas y no manejar sino fábulas acerca de ellas (...)"

E) "Los niños ven en la pantalla escenas de sexo y matanzas bélicas (...)"

Clave: A

\footnotetext{
${ }^{6}$ Ver texto y preguntas en "Prueba oficial de Lenguaje y Comunicación", pp. IO-II, disponible en: https://psu. demre.cl/publicaciones/listado-2OI3 [Consulta 2O-II-2OI8].
} 
Para responder correctamente el ítem se requiere experticia previa en la lectura de texto argumentativo, pues el prejuicio aparece dentro del desarrollo de una prosa donde se argumenta en torno a la influencia de la televisión. Los datos muestran que resultó bastante difícil de abordar: solo el I5,2\% dio con la clave y el 5\%,2\% prefirió omitir.

\section{Ejemplo 5: determinación de la perspectiva del emisor frente al tema central del texto}

Texto estímulo: fragmento de "Prólogo a Historia del Ingenioso Hidalgo Don Quijote de La Mancha”, edición 2005 (M. Vargas Llosa), 424 palabras, prueba 2OI2, texto $9^{7}$. En el texto se analiza la ficción como tema central en la novela de Cervantes y sus repercusiones en la novelística posterior.

El ítem 77, referido a la habilidad de analizar-interpretar, evalúa si el estudiante es capaz de determinar la actitud del emisor hacia el tema central del texto, a partir de marcas textuales que den cuenta de esta actitud. Es decir, no solo es necesario saber de qué se habla el texto y cómo se organizan las ideas, sino extraer la perspectiva discursiva predominante.

\section{El texto de Mario Vargas Llosa presenta una perspectiva discursiva}

I. objetiva, pues se limita a informar, evitando opiniones que manifiesten su posición frente a lo expuesto.

II. admirativa, porque rescata aspectos positivos tanto del personaje como del autor de la novela.

III. crítica, ya que enmarca su pensamiento en una reflexión, para influir en el receptor con sus ideas.
A) Sólo I
B) Sólo III
C) Sólo I y II
D) Sólo I y III
E) Sólo II y III

Clave: E

Dado que el ítem es de doble disyunción ${ }^{8}$, se presentan solo tres perspectivas posibles, siendo admirativa y crítica las correctas, puesto que el emisor exhibe una actitud favorable hacia el Quijote, por ejemplo, cuando destaca el aporte que significó a la narrativa ("los novelistas contemporáneos [...] son todos deudores del Quijote") y a través de

\footnotetext{
7 Ver texto y preguntas en “Prueba de Lenguaje y Comunicación”, pp. 6-9, disponible en: https://psu.demre.cl/ publicaciones/listado-20I4 [Consulta 2O-II-2OI8].

${ }^{8}$ Es decir, un tipo de ítem que requiere por parte del estudiante dos procesos de comprensión sucesivos: primero, la determinación de la exactitud o inexactitud de los enunciados numerados del uno (I) al tres romanos (III), y, en segundo lugar, la selección de la opción que presenta la identificación y/o combinación de tales enunciados formulados como la opción correcta.
} 
una argumentación crítica que busca reafirmar su vigencia y su valor literario en la actualidad. El buen lector debería ser capaz de darse cuenta de esta perspectiva y descartar que se trata de una perspectiva objetiva, porque hay marcas textuales (como calificar de "innovadora" la forma narrativa de la novela, en el párrafo cuatro) que evidencian una tendencia laudatoria por parte del emisor hacia el tema que desarrolla. En su aplicación, el ítem resultó difícil $(22,3 \%)$ y presentó una elevada omisión $(54,3 \%)$.

\section{Ejemplo 6: síntesis de la idea principal}

Texto estímulo: fragmento de Elábaco, la lira y la rosa. Las regiones del conocimiento (José Luis Díaz), 488 palabras, prueba 20I2, texto $7^{9}$. Se trata de un texto expositivo, en el cual se realiza una comparación entre el proceso creativo propio del arte, con el proceso de descubrimiento por parte del científico.

El ítem 62 evalúa la habilidad cognitiva de sintetizar globalmente, es decir, solicita la determinación de la idea principal del texto, para lo cual se entrega una parte de esta en el enunciado (se trata de la semejanzas entre dos ideas presentadas en el fragmento).

62. En su globalidad, el fragmento expone las semejanzas entre la

A) delicadeza del artista y la observación del científico.

B) creatividad de un artista y la rigurosidad del científico.

C) exposición de una obra de arte y una evidencia científica.

D) transformación de formas en el arte y la verificación de datos científicos.

E) producción de una obra de arte y el proceso de una investigación científica.

Clave: E

El ítem resultó difícil, estadísticamente hablando (33,1\%), y presentó una elevada omisión $(40,9 \%)$, cifras que indican que la síntesis de la idea principal no resultó clara en este caso, situación que puede explicarse por el hecho de que en el texto se desarrollan varios temas bastante abstractos: el sentido de la obra de arte, el rol del científico, el método propio de la ciencia, el papel de la observación en el arte; además de estructurarse a través de un contraste entre ciencia y arte.

\footnotetext{
${ }^{9}$ Ver texto y preguntas en "Prueba de Lenguaje y Comunicación”, pp. I2-I3, disponible en: https://psu.demre.cl/
} publicaciones/listado-2OI4 [Consulta 2I-II-2OIO]. 


\section{Conclusiones}

A partir de datos referidos a ocho aplicaciones de la PSU-L, en la investigación se seleccionó un corpus reducido, constituido por textos que presentaban características discursivas semejantes a las lecturas académicas que los estudiantes universitarios enfrentarán desde sus primeros días en la educación superior. La metodología consistió en analizar las estrategias de lectura que estaban implicadas en la resolución de los ítems, los elementos textuales que debían ser considerados para una buena lectura, y el comportamiento estadístico de cada ítem seleccionado (estadígrafos dificultad y omisión obtenidos desde sus aplicaciones en pruebas oficiales). El objetivo final era llegar a conclusiones acerca de las habilidades de los egresados de la educación media en Chile para realizar una lectura comprensiva de textos argumentativos y expositivos, cuyas características discursivas principales son: referirse a algún área del saber que puede ser poco familiar para los estudiantes; focalizarse en contenidos abstractos y en procesos más que en objetos conocidos (primacía de la función referencial por sobre la emotiva o la conativa); haber sido elaborados con una prosa cuidada (registro estándar); y, finalmente, utilizar un léxico culto, amplio y preciso.

Los resultados indican una baja capacidad de los estudiantes para responder adecuadamente ítems que requieren una buena lectura comprensiva de los textos antes caracterizados. En particular, el ejemplo 3 analiza el reconocimiento del patrón textual en un fragmento expositivo; su omisión alta indica desconocimiento de este contenido, aun cuando la organización interna de los textos expositivos es un contenido mínimo obligatorio de II medio (MINEDUC, 2005: 43); la dificultad mediana muestra cierto dominio por parte de quienes sí respondieron el ítem. La síntesis de una idea principal también resultó difícil, lo que se explica al analizar la complejidad y el grado de abstracción del texto estímulo (el cual presenta un contraste entre los atributos del arte y de la ciencia como actividades humanas). Estrategias de lectura que corresponden a fragmentos argumentativos, como determinación de la perspectiva del emisor frente al tema que trata (ejemplo 5), reconocimiento de un prejuicio que se encuentra sutilmente deslizado en la presentación del tema (ejemplo 4) o identificación de la tesis (ejemplo 2), evidencian alta dificultad (inferior al $40 \%$ y, en algunos casos, al $20 \%$ ) y omisión elevada (superior al 30\%). Estos resultados demuestran que las habilidades de lectura que implican obtención de información no explícita, a través de la realización de interpretaciones, síntesis e inferencias, por ejemplo, no han sido adecuadamente adquiridas en la etapa escolar. Dada la propiedad de "referencia curricular" que caracteriza a la PSU como instrumento de evaluación, es posible afirmar que este deficiente nivel de comprensión lectora detectado en los estudiantes está asociado, además, a un deficiente conocimiento y manejo de estructuras textuales que formaban parte de los contenidos mínimos obligatorios en el periodo 2005-20I2, pues el análisis realizado consideró aspectos teóricos del discurso expositivo (abordado en el nivel de II medio) y del discurso argumentativo (III medio). 
En este sentido, los resultados de la investigación son coincidentes con otras investigaciones realizadas en torno a habilidades comunicativas, que plantean la existencia de serias deficiencias en los estudiantes que acceden a la educación superior, para leer y escribir en las disciplinas; lo que sin duda corresponde a un desafío aún mayor, pues lo que aquí se ha demostrado es que la lectura de textos apropiados para el último nivel de enseñanza media presenta carencias, las cuales, además, se hacen extensivas a la etapa inicial de formación universitaria. 


\section{Referencias bibliográficas}

-Aiken, L. R. (I99I). Psychological Testing and Assessment. Massachusetts: Allyn \& Bacon.

- Álvarez, T. (I996). El texto expositivo-explicativo: su superestructura y características textuales. Didáctica, 8, 29-44.

- Bassols, M. y A. M. Torrent. (2OI2). Modelos textuales. Barcelona: Octaedro.

- Bisquerra, R. (Coord.) (20I4). Metodología de la investigación educativa. Madrid: La Muralla.

- Bolívar, A.; R. Beke y M. Shiro. (20IO). Las marcas lingüísticas del posicionamiento en las disciplinas: Estructuras, voces y perspectivas discursivas. En Parodi, G. (Ed.). Alfabetización académica y profesional en el siglo XXI: leer y escribir desde las disciplinas. Santiago de Chile: Planeta, 95-I25.

- Bono, A. y S. De la Barrera. (1998). La producción de escritos académicos de los estudiantes universitarios. Análisis de aspectos conceptuales y estratégicos. Revista Con-textos de Educación, I(I), I3O-I37.

- Braslavsky, B. (2003). ¿Qué se entiende por alfabetización? Lectura y vida, 24(2), s/p. Obtenido el 20 de diciembre de 2018 desde http://www.lecturayvida.fahce.unlp.edu.ar/ numeros/a24n2/24_O2_Braslavsky.pdf.

- Cabrera, M. y S. Caruman. (20i8). Relación entre tipo de texto y comprensión lectora, en una prueba estandarizada chilena. Perfiles educativos, XL (I6I), IO7-I27.

- Calsamiglia, H. y A. Tusón. (2012). Las cosas del decir. $3^{\text {a }}$ ed. Barcelona: Ariel.

- Carlino, P. (2005). Escribir, leer y aprender en la universidad. Buenos Aires: Fondo de Cultura Económica.

- Carlino, P. (20I3). Alfabetización académica diez años después. Revista Mexicana de Investigación Educativa, I8(57), 355-381. Obtenido el I7 de octubre de 2018 desde https:// www.redalyc.org/pdf/I40/I4025774003.pdf.

- Crocker, L. L. y J. Algina (ig86). Introduction to Classical and Modern Test Theory. New York: Holt, Rinehart \& Winston.

- Departamento de Medición, Evaluación y Registro Educacional (DEMRE), Universidad de Chile. (20I7). Prueba de Selección Universitaria. Informe técnico. Proceso de admisión 2017. Obtenido el Io julio de 2017 desde https://psu.demre.cl/estadisticas/documentos/ informes /2OI7-vol-2-informe-piloto-2OI6.pdf. 
- Escarpeta, J.A. (20I9). La influencia de los aspectos socioculturales y lingüísticos en los niveles de literacidad: el caso de alumnos de la Facultad de Idiomas, Univ. Veracruzana. Álabe, I9. [www.revistaalabe.com]. DOI: IO.I5645/Alabe20I9.I9.9.

- Escofet, A.; M. Rubio y L. Tolchinsky. (I999). Escribir en la universidad. Revista Española de Pedagogía, 57 (214), 547-565.

- Hall, B. y M. I. López. (2OII). Discurso académico: manuales universitarios y prácticas pedagógicas. Literatura y lingüústica, 23, I67-I92. Obtenido el 2I de enero de 2019 desde https://scielo.conicyt.cl/pdf/lyl/n23/artio.pdf.

- Marín, M. (2006). Alfabetización académica temprana. Lectura y Vida. Revista latinoamericana de lectura, 27(4), $3^{2}-34$. Obtenido el 20 de octubre de 2018 desde http://www. lecturayvida. fahce.unlp.edu.ar/numeros/a27n4/27_o4_MarIn.pdf.

- Ministerio de Educación (MINEDUC), República de Chile. (I998, 2005 y 2009). Objetivos Fundamentales y Contenidos Mínimos Obligatorios de la Educación Básica y Media. Santiago.

- Narvaja de Arnoux, E.; M. Alvarado; E. Balmayor; M. Di Esefano; M. Pereira y A. Silvestri. (1996). El aprendizaje de la escritura en el ciclo superior. En Z. Solana (Comp.). Adquisición de la escritura. Rosario: Ediciones Juglaría, 199-234.

- Neira, A.; F. Reyes y B. Riffo. (20I5). Experiencia académica y estrategias de comprensión lectora en estudiantes universitarios de primer año. Literatura y Lingü̈́stica, 3I, 22I-244.

- Núñez, P. y C. Espejo. (2005). Estudio exploratorio acerca de la conceptualización del informe escrito en el ámbito académico. En Harvey, A. En torno al discurso, I35-I48. Santiago de Chile: Ediciones Universidad Católica de Chile.

- Roldán, L.A. y V. Zabaleta. (20i6). Lectura y Escritura. Autopercepción del Desempeño en Estudiantes Universitarios. Revista Iberoamericana de Diagnóstico y Evaluación, 42(2), $27^{-38}$.

- Sánchez, S. (20I6). El letrismo académico a través de las asignaturas en las universidades públicas estatales de México. Revista Iberoamericana de Educación Superior, 7(I9), I3I-I5I. Obtenido el 6 de mayo de 2018 de https://www.ries.universia.unam.mx/index.php/ries/ article/view/ıgr. 